\title{
Linx
}

Revue des linguistes de l'université Paris X Nanterre

$60 \mid 2009$

Nonne scripta manent?

\section{Re- dans tous ses états, un « préfixe » marquant l'aspect implicatif}

Isabelle Weill

\section{OpenEdition}

1 Journals

Édition électronique

URL : http://journals.openedition.org/linx/705

DOI : 10.4000/linx.705

ISSN : 2118-9692

Éditeur

Presses universitaires de Paris Nanterre

\section{Édition imprimée}

Date de publication : 1 mai 2009

Pagination : 119-140

ISSN : 0246-8743

\section{Référence électronique}

Isabelle Weill, «Re- dans tous ses états, un « préfixe » marquant l'aspect implicatif », Linx [En ligne], 60 | 2009, mis en ligne le 29 mars 2012, consulté le 17 juin 2020. URL : http://journals.openedition.org/ linx/705; DOl : https://doi.org/10.4000/linx.705 


\title{
$R e$ - dans tous ses états, un « préfixe " marquant l'aspect implicatif
}

\author{
Isabelle Weill \\ Université Paris Onest Nanterre La Défense
}

Pendant les dix-neuf années au cours desquelles j’ai connu Jacques Anis (je n'oublierai jamais tous ces lundis où nous déjeunions ensemble pour nous diriger ensuite vers les salles où il avait cours), nous avons surtout parlé de linguistique et de notre goût commun pour les langues et littératures anciennes et modernes : nous possédions en commun, outre le français à toutes époques, le latin, le grec et l'anglais ; j'apprends en ce moment l'espagnol qu'il dominait bien. Nous discutions de nos recherches et je lui ai fait souvent part de mes angoisses à propos d'un préfixe commun à toutes les langues romanes, auxquelles on peut joindre l'anglais (pour la moitié de son lexique, celle d'origine française), le préfixe $R \boldsymbol{e}$-. Nombre de médiévistes, de linguistes modernes ont étudié cette forme, bizarre à plus d'un titre: on s'imagine que cela veut dire simplement à nouveau, en arrière (ce qui n'est déjà pas la même chose); Rey note une valeur intensive d'achèvement ou une valeur affaiblie qui n'ajoute aucun sens supplémentaire à la base; tous les médiévistes savent que cet élément peut signifier de son côté, à son tour. Jalenques, dans une très intéressante thèse de sémantique consacrée à ce préfixe, constate que les dérivés latins présentent souvent un sens opposé à celui du verbe simple. Or j'ai travaillé il y a quelques années dans un article des Mélanges pour Michel Arrivé, sur les mots de sens opposé et je m'étais promis d'y revenir: $\boldsymbol{R}$ - fait partie de ces éléments : si renier (le renégat, an anglais, renegade) signifie assez logiquement dans tous les stades du français, refuser la croyance, la foi en Dieu, on peut s'étonner de constater qu'en ancien français recroire (recreant, signifiant «mécréant» existe toujours en anglais) peut avoir un sens en 
commun avec le verbe précédent renier, recroire veut dire "refuser de croire en soimême et avouer, après des efforts qui s'avèrent vains, être un vaincu et un lâche indigne d'être chrétien »: nous connaissons tous encore recru «fourbu de fatigue », participe passé de l'ancien recroire, qui marque l'état d'un animal ou d'un homme qui a été audelà des limites où il se sentait capable de poursuivre un effort. Et que penser du verbe remanoir, en ancien français, qui veut dire «demeurer intact et bloqué sur ses assises, subsister fort diminué après une opération de soustraction » mais aussi « disparaitre », se volatiliser, dans le cas des gens dont le destin achève son cours sur le champ de bataille, ou dans le cas de combat, des paroles, des discussions qui ne vont pas plus loin et qui restent sans suite? Et ce genre de phénomène existe aussi en français moderne: si je résous (soudre a disparu du français, mais notre "préfixe», nous le verrons, continue à avoir une vie propre) un problème, il n'y a plus de problème mais si le brouillard se résout en pluie, il y a quelque chose de plus consistant qu'avant; Claudine Normand, une amie comme moi de Jacques Anis, a analysé les problèmes que posait le verbe regretter, dont nous dirons quelques mots, en dépit du fait que morphologiquement on ne puisse parler de préfixe.

Je vais tâcher d'exposer les grandes lignes de ces difficiles recherches dont j'avais présenté les prémisses à notre ami à tous, Jacques Anis et de voir comment ce préfixe permet au sujet d'aller an fond des choses (c'est un des sens de rursus en latin) et de rester présent à l'arrière plan ; nous allons parcourir vingt siècles et quatre langues ou états de langue successifs: le latin, l'ancien français, le français, l'anglais (en notant son influence sur le français récent). Nous verrons que notre "préfixe » permet à l'homme dans la langue, pour citer Benveniste, de regarder de haut le procès du verbe et d'agir sur lui et de le diriger, vers l'arrière, à nouveau, de le déplacer, de le faire rebondir, de l'échanger, de marquer une certaine intentionnalité (qui peut aller jusqu'à la satisfaction complète ou à l'échec programmé, et c'est ce qui a été compris comme un sens intensif), de faire dupliquer le procès par un autre sujet, et si l'on va trop loin au fond des choses, de l'annihiler. Les objets, à travers ce "préfixe ", ne peuvent être manipulés qu'en renvoyant la qualité qui leur est propre, réfléchir pour ce qui brille comme une couleur, la lune, résonner pour un bruit, réverbérer pour la lumière. $R \boldsymbol{e}$ - me paraît se comporter comme un élément sémantique porteur d'une valeur aspectuelle et c'est un « préfixe » que l'on retrouve aussi dans les noms d'action. Tous les chercheurs se sont demandé si n'importe quel verbe était susceptible de recevoir ce genre d'affixe et tous ont constaté qu'il était bien difficile de répondre: je ne suis sûre que d'une seule chose, il doit être rare de préfixer ainsi un verbe dont le sens s'oppose à une quelconque implication du sujet dans le procès comme dans le cas de mourir, périr, faillir, s'opposant à tuer ${ }^{1}$... On peut en revanche renaître.

Nous devons évidemment justifier formellement la catégorie "préfixe» que nous utilisons; il se présente sous trois variantes bien connues $R e / R e ́ / R, R e$ - devant consonne et $R e ́$ devant voyelle dans les créations récentes (on a réaffirmer à une époque qui n'est plus celle de ravoir), ce qui implique que le préfixe tient à manifester son existence en tant que tel; mais peut-on parler de préfixe lorsque la base a disparu du

\footnotetext{
1 Il faut évidemment signaler l'emploi qu'en fait Voltaire dans Candide; le héros, excédé à la fin du roman de voir que le frère de Cunégonde est toujours possédé par les mêmes principes qu'il juge idiots, s'exclame noblement : « je te retuerais, si j'en croyais ma colère. » (Fin du chap. 29, p. 218)
} 
français ou n'a seulement jamais existé de façon indépendante comme dans le cas de regretter (les chercheurs hésitent entre une base germanique ou une base latine) ? Si nous n'avons aucun doute dans le cas de ré-sonner (la forme sonner existe avec un sens proche et la sonorisation du $-s-$ intervocalique n'est pas un problème), nous restons déjà perplexe devant le verbe ré-flécbir car la «base » n'a qu'un sens éloigné de celui du verbe non préfixé. Je voudrais néanmoins souligner que $R \boldsymbol{e}$ - bénéficie d'une certaine indépendance en ancien français où il se comporte comme une particule séparée de la base aux temps composés : il refait, il ra fait; l'élément très pose aussi des problèmes de classement, c'était d'abord un préfixe (Balzac écrit encore trèsbelle) avant de devenir un adverbe à emploi très contraint et le classement de ces éléments mériterait une nouvelle étude approfondie que nous laisserons pour une étude ultérieure. Constatons, avant de passer à l'étude purement sémantique de cette sorte de particule élémentaire, que $R e$ semble doué d'une vie propre, que les verbes précédés de cet élément continuent à changer de sens comme les verbes préfixés : la langue échappe aux catégories créées par la linguistique.

Nous ne verrons pas les sens bien étudiés de en arrière, à nouveau et nous allons donc, en souvenir de Jacques qui a rejoint Michel Galmiche au pays de la linguistique fantastique, entamer un voyage dans l'espace et le temps en remontant d'abord deux mille ans en arrière. Nous suivrons le chemin ouvert par les chercheures en diachronie comme Christiane Marchello-Nizia et Michèle Perret, infiniment plus spécialistes que moi de ces interactions dans la langue. Nous nous contenterons de chercher de façon empirique et forcément très incomplète si l'on peut trouver des régularités et une continuité ou une rupture dans certains effets de notre "préfixe ». Avant d'étudier le préfixe dans la langue origine, le latin (langue où morphologiquement on peut toujours le caractériser comme préfixe) et de passer aux stades suivants, nous essaierons de le caractériser.

\section{$R e$ - et ses implications}

Benveniste a montré que les marques de subjectivité sont plus nombreuses dans les langues anciennes et il a étudié les effets très variés que ces marques peuvent avoir sur les relations de significations. Il a en particulier analysé les effets du moyen en grec ancien (vol. 1, chap. XIV, p. 168-175) et montré qu'un «même verbe, par le jeu des désinences pouvait signifier prendre ou donner ». $R \boldsymbol{e}$ - n'existe pas en grec mais dans cette langue le sujet s'impliquait pour diriger le procès en s'incluant dans l'action grâce au moyen (des formes conjuguées indiquant que l'on fait l'action pour soi); cette " voix » a existé avant le passif et possède des formes communes avec ce dernier, sauf au futur et à l'aoriste. Je citerai un verbe qui sert de paradigme, iémi, « j’envoie», s'opposant au moyen, iémai, «je me dirige dans une direction suivie et choisie donc je m'élance »; pour citer un autre verbe moins courant, aero, à l'actif signifie "prendre en main un objet pour le présenter, l'offrir »; au moyen, "prendre un objet en main pour soi, pour l'obtenir et s'en emparer ». On a donc bien des sens opposés mais dans le cas du moyen, Benveniste note que le sujet est engagé dans le procès, qu'il est intérieur au procès, qu'il en est le siège. L'espagnol connait un emploi de certains verbes à la forme pronominale qui est comparable à la voix moyenne : ir signifie «partir» alors 
qu'irse signifie «s'enfuir, se sauver ». Notre préfixe ne relève néanmoins pas du même domaine, car le procès s'accomplit hors du sujet en obéissant aux contraintes imposées par le préfixe lui-même ; c'est pour cela que nous avons pensé à une valeur aspectuelle, que nous avons hésité à qualifier d'empathique, réactive, interactive, connective avant de choisir, faute de mieux, implicative, le sujet se montrant plus prisonnier du réseau des significations que son véritable organisateur. Nous allons voir que le latin utilise le préfixe $R \boldsymbol{e}$ - pour des effets de sens également fort étonnants ; nous en étudierons trois qui s'enchaînent logiquement en partant du sens de «au fond des choses, à partir de ce fond » qui est un des sens de l'adverbe rursus, sens qui ne figure pas dans les dictionnaires mais que m’a confirmé le seiziémiste (et latiniste) Michel Magnien ; j’ai trouvé dans le Gaffiot, à l'entrée bullio un emploi qui confirme mon hypothése : demersus summa rursus non bullit in unda (Persius Flaccus), "noyé en eau profonde il ne renvoie pas de bulle d'air à la surface».

\section{Re- en latin}

Re- permet d'abord au procès de repartir en sens contraire comme un boomerang qui rebondit en notant une sorte de réaction en chaîne: jacio, "lancer des traits", s'oppose à rejicio, « riposter en lançant des traits contre les attaquants, rejeter». Nous citerons Cicéron : eorum ferrum et audaciam rejeci in campo ", j'ai repoussé leurs armes et leur audace au champ [de Mars] ». Do veut dire «donner, accorder à quelqu'un »; reddo, c'est "produire en retour en renvoyant à l'expéditeur», voire « rejeter quelque chose devant quelqu'un». Arguo implique que l'on cherche à prouver son droit devant un tribunal et avec redarguo, on se défend en avançant des arguments en réplique pour prouver ses erreurs à qui veut vous voir inculpé. Clamo signifie qu'on pousse des cris pour s'exprimer hautement et affirmer légitimement les choses : tabulae praedam illam istius fuisse clamant (Cicéron), « les registres 'crient/proclament' que c'était là le butin de cet individu [Verrès] »; avec reclamo, on proteste en faisant valoir un point de vue qui s'oppose au point de vue précédent; on retrouve cela dans les verbes anglais to claim et to reclaim et je pense que si on revendique en anglais la possession d'un bien, une concession aurifère (a claim est une "concession", « un terrain aurifère sur lequel le chercheur a seul des droits »), un trône, on a intérêt à employer le verbe simple car personne n'a proclamé ses droits sur l'objet en question auparavant et on doit avoir plus de légitimité en la matière.

Si on va trop au fond des choses par un mouvement rétrograde, tout peut devenir négatif et le verbe base et le verbe préfixé peuvent avoir des sens opposés ; cedo signifie «avancer, ne pas résister», et donc "aboutir à quelque chose » : hoc quoque ad tuam gloriam cedet, " cela réussit à ta gloire » (Tacite). Recedo veut d'abord dire « se retirer par une marche arrière, s'éloigner du point origine »: Anchisae domus recessit, la maison d'Anchise est retirée, dans un endroit reculé (Virgile) ; puis le mouvement d'éloignement s'accentue et on aboutit au sens de «disparaitre, se perdre, tourner à néant»: nomen hostis a peregrino recessit (Cicéron), "le terme " hostis » a perdu son sens originel d'étranger »; maris ira recessit (Ovide), « la colère de la mer s'évanouit». On a donc le sens du verbe simple qui s'oppose à celui du verbe préfixé (aboutir et disparaitre) alors 
que le verbe préfixé oppose lui-même les sens de s'éloigner et disparaitre. Restringo contient aussi deux sens opposés, d'abord celui de « serrer, ramener en serrant vers soi » (stringo veut dire pincer, serrer) puis « desserrer, ouvrir». Claudo, «fermer» s'oppose à recludo qui signifie "fermer et ouvrir». Velo et revelo s'opposent de la même façon, avec « voiler et dévoiler» pour le composé ; dans ce cas, la morphologie n'a pas conservé cette intéressante opposition en français (révéler) et pas plus en anglais (to reveal) où les deux verbes ne sont pas morphologiquement préfixés, ne sont jamais ressentis comme tels, notre particule $R \boldsymbol{e}$ - ayant cessé d'avoir une vie indépendante. Condo veut dire " placer dans un endroit sûr, fonder » (ut conderet urbem, au début de l'Enéide) alors que recondo passe du sens de «mettre à sa place primitive» à celui de « faire disparaittre définitivement au fond des choses» : aves avido recondidit ore (Ovide), "il engloutit les oiseaux dans sa gueule avide ». Nuntio signifie « faire savoir » et renuntio, « annoncer en retour, se dédire, marquer un arrêt » : renuntiare alicui hospitium (Cicéron) « annoncer une rupture des liens d'hospitalité ». Moveo a pour sens « mettre en mouvement, provoquer, faire se produire »: on peut citer movere risum(Cicéron), " provoquer le rire » ou movere numen (Tite-Live), "manifester sa puissance »; mais removeo, c'est « écarter, éloigner» et à la limite «faire disparaître » : removere aliquid de medio (Cicéron), "faire disparaittre quelque chose » et il est curieux de noter que l'anglais to remove (la base move est bien attestée) présente exactement la même palette de sens. Pono signifie «placer, établir solidement » : Roma in montibus posita (Cicéron), «Rome établie/installée sur des monts »; mais repono implique un retour en arrière allant jusqu'à l'idée de «mettre de côté en gardant en réserve » : arma reposita (César) : « les armes mises à l'écart ».

On a un troisième type de sens qui consiste à continuer dans le même sens le mouvement primitif en l'amplifiant pour le poursuivre au fond des choses, en s'y impliquant (c'est là la "valeur intensive » des dictionnaires) ou en compensant un mouvement d'ordre détrimentaire, suivant le cas. Bullio veut dire « bouillir, envoyer des bulles d'air à la surface à partir du fond » et rebullio, « bouillir » aussi, mais « en faisant déborder à l'extérieur et en rejetant les bulles à partir du fond». Cubo signifie «être couché » et recumbo, «s'affaisser en s'écroulant». Caedo veut dire " couper» face à recido, « retrancher, réduire, diminuer ». Maneo signifie « rester intact», alors que remaneo note le fait de rester intact après une soustraction : pars remanebat integra (César et Cicéron); nous retrouverons ce sens en ancien français (remanoir) et en anglais (to remain, a remain, a remnant). Censeo, signifie « estimer, évaluer»; mais avec le préfixe contenu dans recenseo, on note que le sujet s'implique personnellement dans l'action et qu'il passe tout en revue, en faisant un examen critique, phénomène qu'on retrouvera encore en ancien français avec par exemple garder et regarder et en anglais avec to collect et to recollect, et (avec le préfixe latin utilisé pour un verbe anglais d'une autre origine que romane) to mind et to remind. De même querre/querir et requerre/requerir sont annoncés par quaero, «chercher à savoir, à se procurer» et requero, "être en quête d'un solution à une question épineuse, rechercher quelque chose qui manque et dont on a besoin ». Capio a pour signification "prendre, saisir» et recipio, "prendre en tirant hors de quelque chose », et donc «accepter, accueillir»; pour ce dernier emploi, le Gaffiot note que « le sens de la particule est effacé », considérant ainsi que, si on ne peut pas classer $R \boldsymbol{e}$ sous la rubrique des sens enregistrés « en arrière, à nouveau », la dite particule ne peut avoir de sens ; je voudrais prouver que Re- a toujours un sens même si ce sens n'est 
pas de prime abord perçu par les locuteurs, ce qui n'a rien de rare en sémantique. Par exemple, le sens de compenser se trouve dans le latin chrétien redimere : emo a pour signification «acheter » et redimo, " racheter une chose vendue » (au sens de mouvement en retour), mais en vocabulaire ecclésiastique (les images fondées sur les valeurs de l'argent y sont fréquentes), racheter un péché implique l'idée de le compenser et de le faire disparaître; comme rédimer n'a guère d'existence, on emploie racheter avec ce sens qui figure dans l'expression le peuple des rachetés, expression (peu claire désormais) signifiant «ceux pour qui le péché (originel) a été compensé par un sacrifice conçu comme une sorte de rançon $»^{2}$. Cette suite de régularités et d'oppositions va se retrouver en ancien français mais comme cet état de langue se caractérise par des traits qui signalent "l'homme dans la langue », selon l'expression de Benveniste, et une sorte de vie personnelle accordée à tout ce qui touche le sujet, les sens de nos verbes "préfixés » vont continuer à diverger.

\section{$R e$ - en ancien français}

Les marqueurs énonciatifs sont très nombreux dans cet état de langue.

J'ai montré dans un article de Linx (Weill, 1995) comment la langue met en scène des éléments physiologiques comme la chair, la peau, le cœur, les yeux, etc. associés à un pronom personnel régime indirect représentant le possesseur du corps en question (il nous reste, en séquences figées : le cour lui manque, la peau lui pèle, les yeux lui piquent mais nous ne pouvons plus dire :*les cheveux lui hérissent, *le pied lui pourrit); on obtient des séquences très fréquentes, surtout dans les textes épiques et satiriques, comme la char li est nercie, "son teint pâlit 》 (Renaut de Montauban, v. 5935); li sans li est muez, « son sang s'arrête de circuler »; (v. 4497) ; tant portent lor haubers a lor char nu a nu I que tres parmi les mailles lor est li peuz issu, «ils portent leurs hauberts sur leur chair nue en sorte que leurs poils sont passés directement à travers les mailles de fer » (v. 14871488).

On connait aussi les emplois des démonstratifs (déterminants, pronoms, adverbes) qui font partie d'un système de repérage très complexe dans l'espace temps de l'énonciation et qui peuvent fonctionner de façon autoréférentielle. Je renvoie en particulier aux travaux de Michelle Perret sur la question.

Le verbe a une place importante et occupe régulièrement la deuxième position dans la phrase (le français est alors une langue dite V2) et, grâce à ses marques de personne, il pallie parfaitement la non expression d'un sujet pronominal.

En outre les survivances du vocabulaire germanique notant des échanges (le don et le guerdon, le don dû en retour) ont pu également favoriser un préfixe qui permettait de mettre en relation des éléments nominaux et des verbes.

Commençons par analyser les rapports entre garder (au sens de « jeter un œil sur quelque chose ») et regarder, « porter son attention sur »... Comme Jalenques (Jalenques :

\footnotetext{
${ }^{2}$ Dans les textes médiévaux, on peut refuser à un ennemi vaincu de racheter sa liberté en demandant sa tête en guise de rançon, ce qui prouve que dans les mentalités, le prix à payer n'est pas forcément de l'argent.
} 
51 sq.) a constaté que dans les deux cas la traduction (qui se veut loin du mot à mot) donnait «regarder» et il en a conclu, disons un peu hâtivement, en confondant les mots et leurs traductions dans une autre langue, que les deux mots étaient synonymes. Jalenques cite un passage de la Chanson de Roland: Oliver est muntet desus un pin balçur, / guardet sur destre par mi un val herbus. / Si vait venir cele gent paienur ; "Olivier est monté sur un grand pin, il regarde à droite au milieu d'un vallon couvert d'herbes et voit venir cette engeance païenne ». Pour comprendre, il faut avoir lu et compris ce qui précède : Olivier fait son travail de second responsable de l'arrière-garde et vérifie s'il n'y a pas des ennemis (il ne devrait pas y en avoir, une trêve a été signée) derrière l'armée. Le narrateur emploie le verbe simple garder, car Olivier ne s'attend pas à voir quelque chose; s'il y avait du préconstruit, le narrateur aurait employé regarder. Lors qu’on regarde de façon préméditée, qu'on regarde éventuellement dans tous les coins d'une pièce, qu'on observe et qu'on calcule la conduite à tenir d'après ce qu'on aura regardé, on trouve le verbe préfixé. C'est pour cela qu'on indique comme sens de ce verbe : « regarder attentivement partout, se retourner pour voir». Il est évident que lorsqu'un homme est poursuivi par des guerriers armés de lances, il se retourne et évalue la situation, il calcule si son avance est suffisante pour lui permettre de s'échapper sans courir le risque d'être embroché dans le dos; dans le cas contraire, il calcule s'il a la place pour faire demi-tour et se battre. Avoir regart de signifie « avoir des raisons pour craindre, surveiller ses arrières »: dans le Roman de Renart, Noble le lion envoie Brun l'ours comme ambassadeur auprès du goupil et lui annonce : vous n'averez de lui regart (branche du Plait, I, v. 451), ce en quoi il se trompe lourdement.

Requerir est au contraire un verbe étonnant par ses emplois polysémiques bien connus et très bien attestés: le sens de base est «chercher à atteindre quelqu'un, quelque chose, en comblant les intérêts et les attentes du sujet grammatical impliqué »; l'action décrite dépend de ce que l'on recherche et de la personne que l'on cherche à atteindre. Si l'on s'adresse à Dieu, à un saint, cela signifie prier. Si on s'adresse à une femme, on lui demande son amour; dans le Lai de Lanval, la reine se plaint faussement d'avoir été déshonorée par le héros : de drüerie la requist (v. 39). On peut employer le verbe pour une demande instante, sans concession possible : on requiert son droit en justice. Mais au cours d'une bataille requerir un ennemi, c'est l'attaquer violemment et lui faire son affaire

Remanoir, un verbe extrêmement fréquent aussi, offre également des sens opposés. Manoir signifie «demeurer chez soi» et remanoir, « rester fidèlement auprès d'un seigneur, d'un roi »; c'est la situation dont se plaint violemment Guillaume au début du Charroi de Nimes car cela ne lui a rien rapporté (v. 120) : il aurait pu aller rejoindre un autre roi qui lui aurait donné un fief (v. 95-101). Le verbe signifie donc « rester bloqué » mais ce qui est bloqué n'évolue plus et peut reculer, voire se volatiliser et disparaître ; une vieille remasue ${ }^{3}$ est retombée en enfance.

On peut par soustraction être laissé volontairement en arrière au cours d'une opération : En .IIII. aguez, sont ça dehors remés/.M. chevaliers garnis et conraez. (le Couronnement de Louis, v. 1563-1564) «mille chevaliers armés et organisés en équipes sont séparés du

\footnotetext{
${ }^{3}$ Raoul de Cambrai traite sa mère de « remasue », v.1036, dans la chanson éponyme ; on voit qu'une demeurée (j'étudierai un jour le "préfixe » de/dé- qui implique une continuité suivie dans le même état) est proche d'une remasue. : remanoir et demeurer présentent d'intéressants rapports.
} 
gros des troupes et placés en embuscade en quatre endroits différents ». Mais un combattant qui remaint sur le champ de bataille est en fait laissé pour mort. Un élément animé (comme un échange de paroles) qui remaint cesse de fonctionner; on dira la bataille remaint, ça n'alla pas plus loin, on en resta là. A la fin de la Chanson de Rolant, les barons sont réunis en conseil pour juger Ganelon ; terrifiés par les menaces de Pinabel, l'impressionnant parent de Ganelon, ils «trahissent » Charlemagne et proposent l'acquittement : Dist l'un a l'altre : "Bien fait a remaneir. / Laissum le plait; « ils se disent l'un à l'autre : il vaut bien mieux s'en tenir là, ne continuons pas le procès »...

Nous allons terminer cette étude trop rapide et incomplète par un verbe bien intéressant, sans précédent en latin, recroire, où le préfixe rend négatif le sens du verbe simple croire : croire signifie essentiellement croire en la vérité de, avoir confiance en ; le sens principal de recroire, extrêmement fréquent dans tous les types de texte, est perdre sa confiance en soi, se fatiguer, se lasser, s'avouer vaincu, ne pas combattre jusqu'à la mort et passer en conséquence pour un lâche; je n'ai jamais vu le sens de croire à nouveau. Au cours d'un combat, on se promet qu'on rendra son adversaire recreant et maté ; Pinabel dans la Chanson de Roland demande à son jeune adversaire, qu'il prétend vouloir épargner, de s'avouer vaincu : Car te recreiz! ( v. 3892). Dans l'Erec de Chrétien (v. 2551), Enide est désolée de devoir annoncer à son mari qui a renoncé à livrer des tournois que tout le monde le considère comme un recreant.

Mais on peut voir aussi s'établir une sorte de valeur d'échange de points de vue et cette idée va nous permettre d'expliquer un passage de la Chanson de Roland, jugé incompréhensible par son dernier éditeur, Cesare Segre, un passage, à mes yeux, où le verbe recroire n'a plus le sens de « renoncer à prouver ses allégations en s'avouant vaincu » mais de "permettre à un autre de pouvoir prouver la vérité de ses allégations». D’abord citons trois vers du Roman de la Rose (vv. 14181-14183) où un emploi de recroire a été relevé dans le glossaire très complet de Felix Lecoy : ...mes face tant que cil recroie,/ por ce que d'amer ne recroie, / qu'el veille autre ami porchacier... " mais qu'elle fasse en sorte que l'autre croie de son côté - afin qu'il ne se lasse pas de l'amour - qu'elle cherche à se procurer un autre ami ». Avec donc ce sens de «croire pour son compte, quant à soi (une chose parfois fausse)», je voudrais ajouter un sens qui me parait relever du vocabulaire juridique ${ }^{4}$ : accepter la parole d'autrui (qui parait de prime abord douteuse ou fausse, ou qui s'oppose à une autre parole) comme une vérité ; il peut s'agir d'une affirmation, d'un serment solennel, ou comme symbole de cette parole et de celui qui la prononce, d'un gage comme un gant ${ }^{5}$, avant un duel judiciaire.

Nous trouvons le premier cas dans Garin le Loherenc. Manuel Galopin, un pilier de taverne quelque peu magicien, raconte devant Begon une histoire assez invraisemblable (appréciation que m'a confirmée Jean-Charles Herbin, spécialiste reconnu de ce type de textes) en affirmant de façon peu claire qu'il est le fils aîné du comte de Clermont

\footnotetext{
${ }^{4}$ Les textes juridiques ne nous sont d'aucun secours car ils sont en latin et le verbe n'existe pas en latin ; il faudrait tomber sur une citation en ancien français (les serments sont toujours prononcés et cités en langue vernaculaire, sous peine de nullité ; on en a un exemple célèbre avec les Serments de Strasbourg).

5 Dans le Charroi de Nîmes, le roi Louis remet son gant à Guillaume et ses neveux comme symbole et de la promesse du don et du don lui-même des territoires sarrasins qu'a demandés le comte.
} 
réduit par son cadet à une vie de débauche; Respont Begon: certes ce poise mie, /-car te recroi, que tu es mes cousins -... L'éditrice, Anne Iker-Gittleman, qui a vu le problème, note dans le glossaire (v. 7281) : « je te crois à mon tour »; cette traduction, présentée ainsi, est impossible car il aurait fallu que quelqu'un d'autre, dans le passage, croit avant cela en la parole de Galopin et je pense qu'il faut traduire : «je suis désolé de ta situation et si tu le dis, ça doit être vrai,/ j'accepte ta vérité, car tu es mon cousin ».

On trouve une affirmation de vérité garantie par un serment, dans le Siège de Barbastre, dans l'édition moderne de Bernard Guidot: nous ne sommes pas vraiment dans le monde sérieux de l'épopée traditionnelle et Girart qui a accepté d'écouter et de suivre un "païen» Malaquin, au cours de ses rencontres amoureuses avec une belle Sarrasine, se demande soudain s'il n'a pas été trahi ; mais Malaquin l'assure qu'il n'en est rien: «Sire, dist Malaquins, sor sains le vous jurron. / Se vous volés juise, loiaument le feron, / que n'i avra boisdie ne nulle mesprison: / Je le vos affie bien sor la loy de Mahom ./-Amis, ce dist Gyrars, nous le vos recreon ». "Seigneur, dit Malaquin, nous vous le jurerons sur des reliques. Si vous exigez une ordalie, nous agirons avec loyauté en sorte qu'il n'y aura ni ruse ni acte de violence. Je vous le garantis sur la valeur de la religion de Mahomet Ami, dit Girart, nous nous en remettons à vous/nous vous faisons confiance, devant un serment de cette valeur. » (la traduction de recroire vient du glossaire de Guidot ; le pronom régime direct le représente le serment agréé, un élément toujours d'ordre religieux).

Après ces deux textes quasi parodiques (Bégon sait bien que son ivrogne de « cousin » affabule; il est comique d'accorder sa confiance à un serment présenté selon des règles strictes mais au nom d'une religion non chrétienne), nous abordons le point où deux vérités s'opposent de façon dramatique avec la chanson de geste d'Ami et Amile : le traitre Hardré accuse Amile (et il n'a d'ailleurs pas tort) d'avoir couché avec Bellissent, la fille de Charlemagne; l'empereur, furieux du déshonneur de sa fille, accepte le gage de Hardré lorsque soixante des membres du lignage se présentent comme «otages» (ce sont des gens qui garantissent, sur leurs biens, sur leur vie, la présence du combattant le jour du duel judiciaire et jugent et attestent qu'il dit la vérité). Amile, qui n'avoue pas les faits mais qui n'en dit rien non plus, a déclaré être prêt à se battre et doit chercher des otages à son tour (vv. 777-778): : Seignor, dist-il, franc chevalier mirable, /envers le roi me recreez mon guaige. »L'éditeur Dembowski traduit dans le glossaire par «donner caution», ce qui est en gros le sens car il est impossible de donner une traduction mot à mot; en effet ici recroire a un sens factitif: Amile demande à ses amis de lui donner l'occasion de garantir sa parole (symbolisée par le gage, complément direct du verbe recroire), de lui permettre de présenter sa requête, de faire en sorte, en acceptant de devenir des otages, qu'il puisse soutenir sa vérité à lui devant Charles (envers le roi) par un duel judiciaire ; seulement les «amis », qui doivent savoir ce qu'il en est, ne sont pas d'accord pour risquer leurs biens voire leurs vies, et se taisent ; Charlemagne refuse alors au héros l'autorisation de soutenir son droit et veut l'exécuter sur le champ, jusqu'à ce que la reine elle-même s'offre comme otage ainsi que sa fille.

Nous en arrivons au procès de Ganelon dans la Chanson de Roland pour retrouver le même vocabulaire et nous allons pouvoir apporter une réponse à un problème qui date des premières éditions du texte au XIX ${ }^{e}$ siècle. Le jeune et frêle Thierri accuse Ganelon de trahison, s'opposant aux conseillers du roi qui préfèreraient 
que Charles accorde son pardon au traître ; aussitôt Pinabel, le gigantesque parent de Ganelon dont il se proclame le champion, remet dans les mains du roi son gant en peau de cerf ; Charles lui demande des pleges (" plegier ", c'est donner un aval comme to pledge en anglais) et trente parents du lignage de Ganelon se présentent aussitôt ; ço dist li reis : «E jo.l vos recrerai.(v. 3848) ». Tierri présente aussi son gant droit à Charles, et $l i$ emperere li recreit par hostage (v. 3852). Ces deux emplois de recroire (le même verbe est employé à propos du coupable et du champion du roi) ont laissés perplexes tous les traducteurs Il se trouve qu'un des sens de recroire en moyen français (largement attesté dans les chartes au XIVe siècle d'après les exemples cités dans la base Lexilogos reprenant les dictionnaires Godefroi et le FEW) est : « renoncer à ses droits de poursuite et remettre un prisonnier en liberté ». Tous les éditeurs traducteurs au complet suivent la très ancienne édition bilingue de Joseph Bédier qui a dû connaitre ces emplois : « il remet Ganelon en liberté sous la caution de ses parents (vos) »; (et avec moins d'unanimité) «il remet Thierri en liberté sous la caution des otages qu'il aura forcément trouvés » (le texte pour une fois ne dit absolument rien sur eux). Segre, dans les notes de son excellente édition, fait observer que la chose n'a aucun sens (le roi ne peut ni ne veut relâcher le traître Ganelon et Thierri n'est pas prisonnier) et qu'en plus dans le deuxième emploi le pronom régime li ne peut être le complément direct du verbe ; certains éditeurs, dit Segre, corrigent même «li recreit » en « le reçeit ». Je dirai personnellement que, pour la deuxième séquence, on peut parfaitement dire que le pronom régime direct a été «écrasé » et qu'on a li pour le li, un phénomène fort banal dans cet état de langue. Nous avons donc un pronom régime direct qui représente, dans chacune des formules rigoureusement parallèles, « le gant » et les pronoms régime indirect, chacun des deux combattants en faveur de qui s'effectue le rite suivi par l'empereur; on ne peut traduire que par «accepter la caution présentée par Pinabel/ Thierri comme garantissant la véracité temporaire de ses affirmations ", ce qui me parait alors fort logique ; et on ne met pas en doute les deux versions des faits mais il est bien entendu que l'un des deux ne dit pas la droite vérité, ce que révèlera le jugement de Dieu. Il s'agit donc à mon avis d'un emploi juridique technique et formulaire de notre verbe (laisser, sous garantie fournie par les otages, momentanément la parole à un accusé qui n'a pas été pris sur le fait, lui permettre d'exprimer à son tour, quant à lui, ce qu'il dit croire être vrai), emploi qui se retrouve bien attesté, nous l'avons vu, dans les chartes où on peut penser qu'on relâche le prisonnier lorsque sa version des faits s'est avérée plausible.

\section{$R e$ en français}

A partir du XVe siècle, on assiste à une disparition d'un nombre conséquent de nos verbes. Christiane Marchello-Nizia constate la diminution des marques relatives à la place de «l'homme dans la langue »; par exemple - l'évolution est achevée seulement au $\mathrm{XVII}$ siècle -, le système complexe des démonstratifs de l'ancien français a disparu au profit d'une opposition déterminants/pronoms, ce qui ne s'est pas fait en espagnol.

Les auteurs de dictionnaires et les médiévistes de la génération qui nous a précédés n'ont pas vu qu'il s'agissait d'un phénomène général et que les valeurs implicatives de Re- allaient aussi subir le même choc: on expliquait autrefois que 
repairier a disparu le jour où on n'a plus compris le sens de "revenir dans sa patrie », issu du latin repatriare: on peut se demander au contraire si un locuteur médiéval quelconque a jamais pu faire cette analyse latiniste. Requerir aurait vu s'affaiblir ses emplois à cause de la concurrence de « rechercher", alors que les sens disparus ne sont pas proches de ceux que possède rechercher. Ce qu'on constate, c'est que le nom d'action correspondant au verbe subsiste et que la langue qui a perdu son statut de langue V2, attachera désormais, à la différence de l'anglais, moins d'importance au verbe et davantage aux noms.

Voyons d'abord deux verbes qui ont perdu leur sens d'origine en laissant un substantif qui l'a conservé : requerir nous a transmis requête. Regarder nous a transmis certains emplois du mot regard (comme avoir droit de regard); pensons aussi au regard, cette ouverture qui permet d'observer « au fond des choses » l'écoulement des eaux usées au pied des maisons et immeubles.

Repairier, rentrer chez soi dans sa maison d'origine, ou dans le camp de base, ou auprès du personnage centre de l'action (je pourrais en citer des centaines d'exemples qui montrent qu'on a toujours été bien loin du latin patria), a bien disparu mais c'était aussi un mot utilisé dans le vocabulaire de la chasse et le repaire a eu d'abord le sens de lieu où l'animal pourchassé veut se réfugier; le verbe est resté en anglais, il est encore dans les dictionnaires, je l'ai trouvé dans des romans anglais sur les guerres maritimes à l'époque napoléoniennes avec le commandement All hands (hand signifie matelot sans grade) repair to the boat, "tous les hommes remontent à bord", mais ce verbe est devenu totalement désuet depuis le début du XXe siècle, m'ont dit mes informatrices. Recorder, « rapporter par écrit son témoignage », n'est plus représenté que par le recors (à l'origine un témoin assistant l'officier de police), l'affreux recors du Mariage de Figaro. Retraire a disparu en laissant retrait, retraite. Revancher n'est plus utilisé, face à la revanche et au simple venger; l'anglais a gardé to revenge, the revenge, pour signifier la vengeance interdite en retour s'opposant à la vengeance de celui qui rétablit la justice, the avenger, un des titres de Dieu dans les textes religieux. Remembrer a laissé le très littéraire remembrance. Plus personne ne voit recroire dans recru de fatigue. Il ne reste de rescorre (arracher au fond des choses ; le verbe simple issu du latin excutere n'a jamais existé en français) que la rescousse ce qui rend très problématique la traduction du roman de Joseph Conrad The rescuer, roman dans lequel l'auteur décline le verbe to rescue, et les noms rescuer, rescue de façon continuelle. Si Jeanne D'Arc n'avait pas existé, nous ignorerions le mot relapse, à moins de connaître l'anglais.

On me dira que ces verbes, à part recroire, n'avaient pas de verbe-base non préfixé pour les soutenir en français mais regretter dont le «préfixe » est bien latin mais dont l'autre élément, probablement un verbe non attesté d'origine germanique signifiant " pleurer» n'a pas disparu et le même désastre est arrivé à des verbes préfixés s'opposant à des verbes simples. Rebouter existait à côté de bouter (Jeanne d'Arc voulait bouter les Anglais hors de France) et voulait dire « repousser en arrière », mais aussi « remettre en place » comme par exemple faire rentrer, en guise de retour à l'envoyeur, des paroles malséantes et des vantardises dans la gorge : Froissart emploie ce $\operatorname{mot}^{6}$ à propos du sort que l'on voulait faire subir à Bayezid, le souverain des Ottomans à la fin du XIVe siècle, lequel

6 Sy prioit le roy de Honguerie par ses lettres au roy de France que il voulsist entendre [...] a resister contre le roy Bassach [...]à ffin que ses vantises luy fuissent ostees et reboutees. Quatrième livre, \47, p.526. 
n'avait eu de cesse d'insulter les chrétiens pour les forcer à « se croiser » contre lui. Il ne nous reste que le rebouteux, ce personnage qui existe toujours dans nos campagnes et qui remet les os à leur place primitive en les repoussant. De remordre pourtant bien vivant chez Rutebeuf ${ }^{7}$, nous n'avons plus que le remords et je ne pense pas qu'on voit facilement le rapport avec mordre. Reclure / reclore ne vit plus que dans le mot reclus et le verbe simple clore (il se confondait morphologiquement avec clouer) a pratiquement disparu aussi. Remanoir me parait le cas le plus dramatique : ce composé extrêmement fréquent de manoir a totalement disparu après le $\mathrm{XV}$ e siècle, en ne laissant pour toute trace que le terme agricole rémanent. Villon emploie encore ce terme dans le Testament: « Et Dieu sauve le remanant», au sens de ce qui subsiste de ses amis sur terre et je me suis toujours demandé s'il n'y avait pas encore un emploi de ce verbe dans le refrain de la Ballade des dames du temps jadis. Rappelons les faits: Villon suppose un interlocuteur fictif chargé de poser des questions sans réponse et le refrain reprend une question elle aussi sans réponse : Mais ou sont les neiges d'antan? Que sont devenues les neiges de l'année dernière? Cette traduction littérale anéantit la poésie des sonorités mais on sait bien que le génie amer de Villon était fort cruel à l'occasion. Le problème se situe dans l'envoi :

Prince, n'enquerez de sepmaine

Ou elles sont, ne de cest an,

Qu'a ce reffrain ne vous remaine :

Mais ou sont les neiges d'antan? (Testament, vv.353-356)

Les plus grands médiévistes (Longnon, Foulet, Lanly, Dufournet) ont édité et traduit ces vers et tous ont affirmé ne pas bien comprendre le sens de remaine. On comprend généralement: "Prince ne cherchez pas de toute une semaine où elles sont, ni de toute cette année, sans qu'à ce refrain je vous ramène... », ou parfois « de peur que le refrain ne vous ramène à cette question ». Je ne vois pas du tout comment on peut ramener quelqu'un à un refrain ou même à une question et on constate que le texte n'est pas sûr : A et F ont pour leçon : "Car ce reffrain le vous remayne (ramaine A) ». Je proposerais de corriger le Qu'a initial en Que, la forme remaine est alors le subjonctif de remanoir, ce qui est même morphologiquement plus plausible. On a alors en traduction: "sans que ce refrain, devant vos yeux, reste/ se dresse bloqué », comme une fin de non -recevoir ; à question idiote (où sont les mortes ?), réponse idiote (où sont les neiges de l'an passé ?), et le poème se clôt sur une saillie sarcastique. On aurait un emploi de nos pronoms personnels à valeur de datif éthique et le verbe remanoir aurait encore le sens de « disparaître faute d'être activé », qu'il avait dans les séquences que nous avons rapidement vues : la joie, la bataille, la parole remaint, ce qui se traduit par: « on en resta là », plus rien ne se passa après.

Nos verbes restants vont toutefois repartir pour un nouveau destin avec le renouvellement du vocabulaire religieux au XVII siècle. On impose aux pénitents de faire une sorte d'introspection en trouvant du positif en eux-mêmes, en leur interdisant de se tourner vers l'extérieur: Rentre en toi-même, Octave et cesse de te plaindre, dit Auguste

\footnotetext{
${ }^{7}$ La mort [... . mort / seux dont le siecle fait remordre, « la mort mord ceux pour qui le monde souffre au fond des choses » De Monseigneur Ancel de l'Isle, p. 96.
} 
dans Cinna. Des verbes d'action vont donc voir leur procès prendre une direction inversée, en rentrant vers l'intérieur, pour servir positivement leurs sujets ou pour leur faire honte et on va trouver à nouveau des verbes de sens opposé ou présentant de curieuses particularités sémantiques. Revenir prend ce type de sens intérieur à partir du XVII e siècle : « revenir à soi » veut dire «reprendre ses esprits»; le verbe sera positif aussi sur le plan matériel : faire revenir de la viande, des légumes, c'est leur donner, sur le feu, une couleur appétissante qu'ils n'ont jamais eue avant. D'autres verbes vont simplifier leurs sens : recueillir qui signifiait «accueillir bien ou mal les invités/les ennemis » (devant une bonne table ou les armes à la main), va devenir entièrement positif et n'aura comme sens que se tourner vers soi-même et ne plus regarder l'extérieur, ou encore rassembler pour soi des éléments extérieurs; rencontrer (à part en boxe, mais on a choisi son destin et les autres rencontres sportives sont moins dangereuses) a perdu pratiquement tous ses emplois militaires et s'est tourné vers l'aspect positif des choses. Requérir perd tous ses sens, devient positif aussi et signifie « demander une chose qui est considérée comme due » (c'est le plus souvent un terme de droit) ou comme exigée pour que tout se déroule dans de bonnes conditions : on parle alors de requérir une peine incompressible, des connaissances, des compétences requises pour tel ou tel type d'actions

D'autres verbes vont prendre plusieurs directions : retourner qui signifiait ou opérer une retraite ou revenir sur le champ de bataille ou rentrer chez soi va connaittre des emplois supplémentaires à partir du moyen français (dès le XIV siècle, dit Rey) : le verbe peut noter un bouleversement complet avec d'abord des sens techniques comme retourner la salade (XVII siècle) ou comme retourner un col, des poignets mousquetaire ou des draps; ces emplois en couture ne sont pas dans les dictionnaires du XIX ${ }^{e}$ et du début du XXe siècle (faits par des hommes peu au fait de ces travaux) alors que le verbe est utilisé avec ce sens dans le Père Goriot de Balzac : « Dès qu'Eugène eut le dos tourné, la vieille courut à sa cuisinière : - Prends les draps retournés, numéro sept. Par Dieu, c'est toujours assez bon pour un mort» (p. 310). Retourner un grand drap usé au milieu consiste à couper tout du long la partie usée au centre, retourner les deux morceaux restants, c'est-à-dire faire basculer les lisières vers le centre et les coudre ensemble finement au point de surjet, tout en faisant des ourlets sur les parties anciennement au centre devenues les lisières; la partie centrale du nouveau petit drap manque évidemment toujours d'agrément. Si on retourne un col ou des poignets, la partie usée à l'endroit, décousue, retournée et recousue, passe à l'envers. Le verbe oscille donc désormais entre deux idées opposées : un bouleversement, une manœuvre de retournement (un emploi connu dans le domaine spatial, et dans l'album Tintin, On a marché sur la lune) et un retour compensatoire vers un état antérieur des choses ou un meilleur état : il est considéré comme positif de retourner un drap (dans le cadre de ce qui fut la bonne économie bourgeoise), de retoumer un espion (pour le bien du service), mais retourner sa veste est honteux, retourner une gifle, un juste retour des choses. Et le bouleversement ne se situe plus seulement au niveau de la réalité : à partir du XIXe siècle, le dictionnaire de Rey note que le verbe marque un bouleversement intérieur, une violente émotion: on se retourne sur son passé, on regarde en soi-même.

Retrowver est bien intéressant aussi et bien compliqué à étudier : si je dis «on a retrouvé des empreintes de dinosaure sur ce chemin rocheux», cela peut vouloir dire qu'on ne les avait pas ni vues ni trouvées auparavant et qu'une recherche plus approfondie les a fait découvrir. Un de mes amis (un peu paranoïaque) a une fois 
intimé l'ordre - à un groupe dont je faisais partie - «je vous retrouve ici» tout en nous quittant et traversant une rue sans autre explication; nous avons cru en toute bonne foi que cela voulait dire: «je vous retrouve ici après que vous avez fait vos achats » donc un sens proche non pas seulement de " en arrière » (car il ne nous a pas trouvés avant) mais de marque d'intérêt pour la situation antérieure à rétablir ; mais notre ami voulait en fait dire «Vous êtes là où vous vous trouvez en ce moment, je vous veux ici bloqués à m'attendre jusqu'à mon retour » (comme pour le verbe remanoir en ancien français) ; j'ai déduit ces sens après qu'il s'est assez violemment plaint : «je suis revenu au bout de même pas trois minutes et vous n'étiez plus là ».

Relever connaît aussi plusieurs emplois ; si on relève quelqu'un de ses fonctions, c'est négatif et c'est une perte ; si on relève une fonction, un titre qui était tombé, un nom qui n'était plus représenté (le sens mélioratif date d'après Rey du XVIe siècle) on fait monter tout cela en honneur, si on relève une sauce ou un plat, on en améliore le goût; si on relève des mailles sur le bas d'un tricot, cela permet de faire une jolie bordure ; si on relève des soldats (pensons à la formule mythique la relève pendant la guerre des tranchées), on les échange contre leurs équivalents; si on relève des notes sur l'ordinateur, c'est encore un simple transfert; si on relève un prix, des exigences, c'est positif et c'est un gain pour certains. Le même verbe signifie donc à la fois gagner, rester tel quel et perdre.

Résigner connaîtra aussi après le XVI siècle deux emplois opposés : de l'ancien français au français moderne, on résigne une charge (je n'ai trouvé nulle part le rapport avec le verbe signer : signe-t-on sa démission après avoir signé une acceptation ?) ; mais aussi, et c'est un sens issu de la théologie protestante, on se résigne à son sort pour obéir à Dieu ; donc on refuse ou on accepte, suivant le type de complément qui suit le verbe.

Je pense que le verbe regretter (il est trop intéressant pour que des raisons formelles me fassent renoncer à en parler) étudié par Claudine Normand se situe dans ce contexte: en ancien français regretter signifie "déplorer la mort d'un être cher», "pleurer sur une séparation, mais parfois aussi déplorer une simple absence due à une série d'événements » : Orson de Beauvais, héros d'une chanson éponyme, dans sa prison et terre sarrasine, regrete Beauvais et dans Renaut de Montauban, chanson où les frères et cousins sont souvent séparés dans les combats, Mangis regrete Renaut, Renaut regrete Mangis.., Renaut regrete Bayard (son cheval aux dons magiques) et c'est ce type d'emploi qui donnera le deuxième sens du verbe actuel. En français moderne, en suivant l'analyse extrêmement fine de notre collègue et amie Claudine, suivant le type de complément $^{8}$, regretter veut dire qu'on aurait voulu qu'une chose n'ait pas eu lieu (le sens date du XVIe siècle) ou qu'on voudrait (au fond des choses, en se retournant vers

\footnotetext{
${ }^{8}$ L'anglais pour opposer deux sens (différents de ceux du français) dans des verbes notant des états mentaux, ne se sert pas d'une différence de complément, mais d'une différence aspectuelle dans la forme verbale même: le present continuous, "forme progressive », insiste sur le fait qu'on vient de réfléchir et qu'on n'aurait pas dû agir ainsi : I regret that the company will have to be sold, "j'ai pris la décision de vendre la compagnie, je l'ai fait à regret, mais c'est fait / J'ai le regret de vous annoncer que ma décision est prise »; I am regretting my decision to give her the job, « je lui ai confié ce travail et je suis de plus en plus conscient que je regrette d'avoir fait ça, je n'aurais pas dû le faire » ((Hewings, Unit1, C).
} 
soi-même, c'est moi qui ajoute ces précisions aux propos de C. Normand) qu'une chose perdue (je ne regrette rien) ait encore lieu. Il y a évidemment des cas où l'interprétation dépend de notre connaissance de la situation extérieure ; si, dans un compte-rendu de procès, on regrette le buis clos, pour interpréter la séquence il faut savoir auparavant si le huis clos a été accepté ou si au contraire, il a été refusé ; la question ne se pose pas si on regrette le passé, car cet élément appartient au monde de ce qui a déjà eu lieu.

Pensons au verbe recaler, en laissant de côté le sens de «caler à nouveau, en arrière ». J'ai lu un article dans Science et Vie (août 2009, N. Ayache) sur la lecture des images médicales acquises avec des modalités différentes: «Nous avons d'abord cru qu'il fallait repérer des points caractéristiques dans chacune des deux images pour guider leur "recalage"; par exemple des lignes saillantes à la surface du cortex pour recaler les images du cerveau »; il s'agit donc de faire coïncider des choses différentes et nouvelles pour bien fonctionner dans la même série, c'est positif; mais si on recale un candidat, on refuse de le faire entrer dans la série et c'est négatif.

Nous avons vu que les interprétations dépendait du type de complément mais il arrive que le sujet (s'il ne s'agit pas de l'animé humain concerné) fasse aussi modifier le sens du verbe; j’ai entendu les deux exemples suivants prononcés par C. Hondelatte, le présentateur de l'émission «Faites entrer l'accusé » sur la chaîne 2 : Le piège se referme : tout fonctionne bien, ce qu'on cherchait porte ses fruits ; la piste se referme, tout va mal, c'est une impasse, ce qu'on cherchait n'aboutit à rien. Rebondir ne veut pas forcément dire « repartir en arrière", il acquiert un sens positif; si je dis l'économie repart, rebondit; c'est positif, mais si je dis le ballon rebondit sur le poteau, c'est la catastrophe. Il y a aussi des cas où il n'y a qu'une différence aspectuelle : je vais me refaire, « TOuT ira bien si on me laisse du temps"; je suis refait, " on m'a eu». Je pense qu'on pourrait faire une recherche générale sur ces types d'emploi opposés en cherchant comment fonctionnent alternativement, dans une sorte de mouvement oscillatoire, la compensation (un des sens principaux en français moderne) et le bouleversement dans un cadre où regretter ne semble plus être une exception aussi notable qu'elle paraissait.

Il y a en fait des quantités d'emploi de verbes préfixés en $R \boldsymbol{e}$ - qui témoignent de ce regard critique et de cette reprise en main du texte (par le texte même) qui constate, après calculs et réflexions diverses, que les événements s'enchaînent dans une sorte de logique immanente; on ne s'étonnera pas de retrouver ici le vocabulaire juridique et journalistique : il a été rattrapé par son passé; il est renvoyé devant un tribunal; les affaires se retrouvent exposées an grand jour; le tribunal rejette la demande de remise en liberté; l'affaire rebondit; ce personnage rebondit. L'interprétation avec le sens de à nouveau, en arrière, est évidemment exclue : être renvoyé devant un tribunal s'oppose même à être renvoyé pour faute grave.

Certains noms présentent également des sens opposés : si on fait une remise de chèques, on donne un objet; si on fait une remise sur un prix, on accepte de moins recevoir et une remise est un garage où on laisse des objets bloqués sans les utiliser.

Nous allons passer à présent à une langue (la moitié de son vocabulaire est d'origine française, ai-je entendu dire au professeur d'anglo-normand, Ian Short) qui a la particularité de faire la part belle aux verbes. 


\section{Re- en anglais}

Le premier mot d'anglais que j'ai appris est la célèbre consigne Remember, la phrase prononcée par Charles I sur l'échafaud dans Vingt ans après: il s'agissait de garder profondément au fond de la mémoire quelque chose de capital pour s'en servir le jour voulu ; l'élément $R \boldsymbol{e}$ - paraît jouer un rôle même si le verbe non préfixé n'existe pas. Je continuerai un jour ce travail en étudiant $R \boldsymbol{e}$ - en moyen anglais, état de langue que je ne domine pas encore assez. Mes exemples ne sont pas pris dans les dictionnaires car ces vingt dernières années, outre des œuvres de Dickens, Jane Austen et Walter Scot, j’ai relu les séries médiévales de Tolkien, lu Dan Simmons, Robin Hobbs, Robert Jordan, George R.R. Martin ; j’ai lu tous les Harry Potter, les récits historiques de Diana Gabaldon et de Bernard Cornwell, les séries d'aventures maritimes à l'époque napoléonienne de J. Forrester et Patrick O' Brien, œuvres pour lesquelles j'ai dû apprendre tout le vocabulaire de la marine à voile ...Je regarde aussi tous mes DVD en version originale anglaise, tout cela en notant depuis plus de quinze ans tous les $\boldsymbol{R} \boldsymbol{e}$ - que je rencontre (j’ai des milliers de relevés) ; tout cela explique que je puisse me sentir aussi capable d'écrire sur de l'anglais que sur du latin. Je me suis néanmoins renseignée, en qui concerne resort en particulier, en prenant comme informatrices deux dames anglaises rencontrées à Bornemouth, et une voisine et amie d'origine australienne, agrégée d'anglais.

L'anglais accorde une importance notoire au verbe dans la phrase, ce qui explique qu'il ait conservé des verbes qui ont disparu du français (to remain, de remanoir; to record de recorder; to repair de repairier, ce dernier ayant souffert de l'homonymie avec to repair, de réparer). N'importe quel nom commun peut devenir un verbe dans cette langue marquée par une absence notable de morphologie, ce qui permet de reconnaitre instantanément le nom d'origine : a wolf est un loup (glouton) et to wolf veut dire engloutir comme un loup, mushroom, le champignon se retrouve dans to mushroom, pousser et s'étendre comme le font les champignons ; a stump est un tronçon, un moignon et to stump signifie aller clopin-clopant. Les verbes s'emploient très facilement de façon diathétiquement neutre : I walk, je me promène; I walk the dog, je promène le chien; I walk the horse, je fais marcher le cheval au pas pour le laisser se refroidir en douceur après un effort soutenu. La distinction actif/passif a des règles très délicates : si on parle de relieved tears, il ne s'agit pas de larmes lâchées mais de larmes exprimant le soulagement (to relieve voulant dire "relâcher, ôter ou soulager »). Les verbes peuvent prendre une valeur factitive: The colorful tapestries on the wall has earned their weavers silver (R. Jordan, The Wheel of time, I) « les brillantes tapisseries sur le mur avaient fait gagner de l'argent aux tisserands qui les avaient faites ». On peut également transformer un verbe en une sorte de nom en lui adjoignant le suffixe -ing, avec le sens de « le fait de ». Je cite une notice informatique (Latex): Eventually you will want to typset a document that requires a more sophisticated formating that you can obtain by informating the two sample imput files; nous emploierions, à la place du verbe en -ing, un nom : par le formatage de... Cette tournure est obligatoire après préposition.

Il faut noter que l'anglais en général n'a conservé que les verbes composés de l'anglo-normand ; manoir, estorer, querre/ querir, membrer, conoistre ont cédé la place devant les correspondants préfixés comme si la langue tenait à conserver la possibilité de jeter un regard sur les choses; la forme encore très française (je ne sais pas comment cela se 
prononce) reconnoitre est toujours employée sur le plan militaire ou naval pour une reconnaissance du terrain mais on peut l'utiliser au sens d'aller à la découverte des choses.

Le préfixe en anglais peut bien sûr avoir le sens de «à nouveau » et dans ce caslà, j'ai constaté que le préfixe était séparé de la base par un trait d'union, comme s'il s'agissait d'une valeur qui n'était vraiment pas la valeur de base : to re-man a man-of-war signifie « réarmer un vaisseau de ligne ». Sinon $\boldsymbol{R} \boldsymbol{e}$ - exprime toujours une certaine exigence de la conscience, un effort violent, et intensifie la forme du verbe simple, lorsqu'elle existe. To require (issu de l'ancien français requérir mais n'ayant pas conservé la forme simple quérir) exprime un ordre auquel on ne peut désobéir : il s'emploie aussi au passif pour des animés humains ; les officiers de marine reçoivent leur lettres d'ordre commençant par You are required to... Il s'agit toujours d'ordre donné par un supérieur à un inférieur et pour exprimer une demande émise par un inférieur, l'anglais a créé to request (un dérivé morphologique du participe du verbe latin quaero) latin comme le montre cet exemple tiré du film Black Hawk down ("La chute du faucon noir », 2002, $\mathrm{R}$. Scott) : un avion survole Mogadiscio en proie à la guerre civile et à la famine «Control order-Command super 64-We got militia shoting civilians at the food distribution centre. Request permission to engage. Over-Super 64, are you tabing fire? -Negative command-Negatif control, U.N.'s juridiction 64. We cannot intervene. Return to base. Over». Le pilote demande la permission (et il tient à l'obtenir) d'intervenir en voyant la milice tirer sur la population civile, permission qui sera refusée. On voit en tout cas que même si les verbes non préfixés n'existent pas, la langue tient à la valeur sémantique du préfixe puisqu'elle s'avère capable de fabriquer un verbe en lui adjoignant et le préfixe et sa valeur habituelle.

To remain (notre ancien verbe remanoir) a perdu, à peu près en même temps que le verbe français, au XIVe siècle, le sens de « disparaître » (je l'ai cherché dans le dictionnaire d'Oxford, mais ce sera ma seule notation diachronique) ; il exprime toujours comme en ancien français une idée de soustraction ou de blocage : her mouth remained open, elle resta la bouche ouverte. Le verbe a aussi le sens de «rester après soustraction »: The gaiety is gone, of course it is, but an atonishing fortitude remains; la gaieté a disparu, c'est normal, mais une étonnante force intérieure se maintient intacte (O'Brian, Blue at the mizzen, «Du Bleu au mât d'artimon»). Trois noms dérivent du verbe, exprimant tous trois l'idée d'un aspect bloqué en suspens : en ce qui concerne remains, il s'agit de restes non identifiables, des ossements humains, des restes de nourriture non mangeables, des ruines ; remnants (le français remanant avec la valeur d'aspect du participe présent) s'emploie par exemple pour un donjon qui se dresse intact au milieu d'un château en ruines, pour des restes de repas réutilisables, et dans la Bible, pour les êtres humains calculés et momentanément épargnés par la colère de l'Eternel ; remainder est le résultat d'une soustraction: on prend dans une boîte des documents, on trie ceux qui présentent de l'intérêt et on replace the remainder dans la boîte

Nous avons vu que l'anglais avait préfixé ainsi des verbes non issus du fonds français, comme to mind et to call ainsi que to reward (gard- et ward viennent de la même racine indo-européenne) avec le sens de "surveiller, regarder», et comme le roi, au cours d'un tournoi, jetait un œil attentif et bienveillant sur le vainqueur, to reward a pris le sens de « récompenser». To mind n'exprime qu'une attention modérée pour un problème posé à la personne considérée, ou que le fait d'associer des choses sans grande importance. Prenons un exemple chez R. Jordan : une des héroïnes Elayne constate 
que ses amies se conduisent comme des fillettes prises en faute; Elayne still was minded of nothing so much as a group of novices having a pillow-fight after Last has tolled when the mistress of novices walked in, "Elayne ne trouvait rien de plus proche, en guise d'association d'idées, que l'image de novices en train de se livrer à une bataille de polochons après que l'heure du coucher a sonné, au moment où la maitresse des novices fait son entrée ». Au contraire si on emploie to remind, on veut signifier (c'est une forme d'intensivité) que quelqu'un a un intérêt personnel très marqué à garder une chose, extérieure à lui, au premier plan de sa conscience, comme le montre cet exemple tiré du film Diamonds are forever, un «James Bond»: "Are you paying attention? May I remind you, 007, that Blofeld's dead. Finished. "M'écoutez-vous attentivement? Puis-je vous demander de garder clairement présent à l'esprit le fait que Blofeld est mort. Terminé ». (C'est moi qui traduis). Le mot reminder désigne une sorte d'avertissement : a reminder of peace est une exhortations à faire la paix Quand on veut appuyer ces dires, on peut insérer $I$ collect ou I recollect: le premier s'emploie pour dire qu'on se réfère à des choses dont on a entendu parler, mais si on emploie $I$ recollect, on fait référence à des connaissances personnelles apprises et assimilées. L'anglais, dans le domaine du rappel à la mémoire, dispose encore de to recall, s'il s'agit d'évoquer en esprit des souvenirs intérieurs mais extérieurs à la situation présente.

Nous terminerons par une étude rapide de to remove; il s'oppose à to move qui signifie simplement bouger, se remuer car notre préfixe, nous l'avons vu, ouvre toutes sortes de portes : le verbe, un peu comme les verbes de l'ancien français, passe de «se déplacer, éloigner, ôter » à éventuellement "assassiner », un effacement plus définitif. Des cousins germains, donc proches, se disent : cousins once removed, et on peut continuer à compter les degrés de parenté ; on emploie ce verbe pour quelqu'un proche de la misère : She was at the edge of her resources, only one step removed from those beggars (R. Jordan); « elle voyait arriver la fin de ses ressources, une seule marche la séparait de ces mendiants ». $\mathrm{Si}$ on est relevé de ses fonctions, on emploie to be removed from office.

\section{Les derniers avatars de $R e$ -}

L'anglais et le français se sont toujours influencés l'un l'autre et un certains nombre de noms d'action correspondant à nos anciens verbes en $R \boldsymbol{e}$ - nous reviendront par l'intermédiaire de l'anglais. Nous avons vu que plusieurs noms dérivés avaient survécu dans la langue classique à la disparition de leurs verbes, et nous allons constater que cette deuxième vague ne nous fera adopter que des noms, ce qui confirme la primauté du nom sur le verbe en français. Et même si le verbe existait encore en français avec un sens affaibli, nous n'avons pas retenu le verbe anglais conservant l'ancien sens, nous n'avons pris que le nom. Nous avons perdu restaurer ${ }^{9}$ au sens de reprendre la place légitimement due, nous n'avons pas réemprunté to restore, mais l'importance de the restoration of king Charles II nous a permis d'introduire la Restauration (1814-1830) en

\footnotetext{
${ }^{9}$ Le titre du roman de Galien li Restauré indique qu'il s'agit d'un jeune homme (un fils qu'a donné Olivier à la fille de l'empereur de Constantinople à la suite d'un pari stupide) qui n'a pas eu par sa naissance tous ses droits légitimes reconnus mais qui les récupère en rejoignant son père à Roncevaux ; le titre est impossible à traduire en français mais il ne pose aucun problème en anglais.
} 
tant que régime politique et période historique et artistique ; c'est néanmoins un terme qui m'a rendue perplexe lorsque j'ai lu pour la première fois Vingt Ans après, car on y voyait que, pour Dumas, ce n'était pas encore un terme figé. Je pense au mot record qui n'a plus en français le sens d'enregistrement (la précision «au fond du cœur» est perdue) mais celui d'exploit sportif; en effet un record non homologué ne sera pas enregistré. Le verbe to revolve et ses dérivés nous a laissé le revolver et à notre époque le crédit revolving qui implique que dès le premier remboursement la capacité à emprunter du consommateur repart à plein et lui permet de réemprunter dans une spirale déroulée sans limite. Le mot rémanence en science nous revient par l'anglais ; pensons aussi à la résilience terme utilisé pour la résistance des métaux passé en psychologie. La descendance la plus étonnante est celle de l'ancien français ressortir, qui signifiait «avancer» ou « reculer/faire » encore avec de nouveaux sens intéressants, étudiés par Michel Arrivé ; des anciens sens, il nous reste le nom ressort qui note encore l'idée d'aller dans tous les sens en rebondissant. J'ai découvert le sens de son correspondant anglais resort en lisant une publicité pour la célèbre station balnéaire de Bournemouth dans laquelle ont séjourné loin du monde et du bruit tous les écrivains anglais de Thomas Hardy à Tolkien : resort désigne un ensemble plein d'agréments, choisi, trié (le verbe simple est to sort out, trier, choisir après un tri, to resort signifiant " recourir à »), créé à l'écart par une communauté humaine dans l'idée de servir de refuge. Dans l'oppressant premier film de Steven Spielberg, Jaws ("Mâchoires », en français «les Dents de la mer»), le maire de l'île explique aux visiteurs du week-end à quel point «Amity Island is a resort» rempli de charme paradisiaque d'autant plus que « amity means friendship »; et il n'est pas besoin d'être un cinéphile pour constater le sens macabre dévolu à "amitié» derrière amity, en pensant au requin qui s'est retranché dans un paradis très personnel et en évoquant aussi le très gore Amityville. Resort est devenu quasiment un mot courant en français pour désigner soit un complexe comme le monde décalé de Disneyworld ou des titres de jeux vidéo sur la Wii se déroulant sur une île ou dans un autre type d'univers clos et reculé.

L'on peut toutefois constater un certain retour de nos verbes; je trouve que restaurer au sens de redonner à un meuble, une habitation son apparence et sa vie d'origine gagne une place dans nos préoccupations. On peut noter un tout nouveau rémaner (on le trouve déjà de fait sous cette forme dans Renaut de Montauban), au sens de persister avec une forme de détermination (et avec un passage dans la conjugaison des verbes à une base et à infinitif vocalique en -er), que j’ai entendu dans la bouche de chercheurs en physique du soleil, des gens qui ont l'habitude de rédiger tous leurs travaux en anglais (un anglais fort romanisé) : ils ont été assez étonnés en constatant que si je connaissais parfaitement le sens qu'ils donnaient au verbe, j'ignorais totalement qu'il était utilisé de façon courante dans leur vocabulaire.

\section{Conclusion}

Une fois terminé notre périple, je noterai en particulier la stabilité de notre préfixe : En diachronie on voit le plus souvent les éléments changer de sens et se figer et il est assez exceptionnel de constater que ce n'est pas le cas. De plus $R \boldsymbol{e}$ - semble exister dans toutes les langues romanes (l'anglais est un cas à part). Même si ce "préfixe » a été 
attaqué à la fin de l'ancien français, on a vu qu'il avait pu reprendre une seconde vie en prenant des valeurs intérieures positives et un emploi comme celui du tout nouveau rémaner est encourageant. Il est quand même étonnant de noter que les locuteurs interrogés ne donnent pour sens à notre préfixe que celui de «à nouveau après avoir été en arrière » (faire et refaire, nouer et renouer) et que les autres significations ne sont pas facilement perçues et encore moins acceptées ; mais on sait que les faits sémantiques (comme la plupart des faits linguistiques) ne sont pas des faits qui s'imposent de prime abord; les significations implicatives ne sont pas non plus aisées à percevoir, surtout lorsque ce n'est pas le sujet grammatical qui commande ces oppositions. J'espère avoir réussi à prouver qu'on peut conférer à notre élément des valeurs aspectuelles plutôt que des valeurs modales. Cette analyse incomplète se veut être une première approche dans une tentative pour trouver des régularités et pour classer ensemble des éléments qui paraissent opaques, épars, exceptionnels, voire dénués de sens et de les rassembler dans une vaste continuité diachronique. 
Re- dans tous ses états, un «préfixe » marquant l'aspect implicatif

\section{REFERENCES BIBLIOGRAPHIQUES}

\section{FRANÇAIS}

\section{Linguistique}

ARRIVE M., 2005, Verbes sages et verbes fous, Lambert-Lucas, Limoges.

Benveniste E., 1966, Problèmes de linguistique générale, 2 vol, Gallimard, Paris.

BURIDANT C., 2000, Grammaire nouvelle de l'ancien français, SEDES, Paris.

JALENQUES P., 2000, Contribution à l'étude du préfixe $R E$ en français contemporain, thèse de doctorat sous la direction de J.J. Franckel, Université Paris 7-Denis Diderot.

LEXILOGos: base privée de données; dictionnaires, en particulier le Godefroi pour l'ancien français.

MarChello-Nizia C., 1995, L'évolution du français, Armand Colin, Paris.

Normand C., 2002, Bouts, brins, bribes, Le Pli, Orléans.

PERret M., 1988, Le signe et la mention, Droz, Genève.

REY A., 1995, Dictionnaire historique de la langue française, Dictionnaires Le Robert, Paris.

WeILL I., 1995, « Le physiologique dans la langue », Linx n 32, p.147-159.

WEILL I., 2002, « Garder et tuer, appuyer et payer, étude linguistique de quatre signifiants lacaniens », in Le Signe et la lettre, textes réunis par J. Anis, A. Eskénazi et J.F. Jeandillou en hommage à Michel Arrivé, L'Harmattan, Limoges, p. 459-475.

\section{Oeuvres citées}

\section{Ancien français}

Bedier J., 1960, éd. et trad. La Chanson de Roland, Piazza, Paris. 214ème éd. depuis 1921.

Chretien de Troyes, 1981, Erec et Enide, éd. M. Roques, CFMA, Champion, Paris.

Froissart J., 2004, Chroniques, livres III et IV, éd P. Ainsworth et A. Varvaro. Lettres Gothiques, LGF, Paris.

Guidot B., 2000, éd. Le siège de Barbastre, CFMA, Champion, Paris.

Guillaume De Lorris et Jean de Meun, 1970, Le Roman de la Rose, éd. F. Lecoy, CFMA, Champion, Paris, 3 vol.

Iker-GitTleman A,. 1996, éd. Garin le Loherenc, CFMA, Champion, Paris.

KAY S., 1996, éd. Raoul de Cambrai, tr. W. Kibler. Lettres Gothiques, LGF, Paris.

LePage Y., 1978, éd. Le Couronnement de Louis, TLF, Droz, Genève.

MCMillan D., 1978, éd. Le Charroi de Nîmes, Klincksieck, Paris. 
Isabelle Weill

Martin J.-P., 2002, éd. Orson de Beauvais, CFMA, Champion, Paris.

RoQues M., 1963, éd. Le Roman de Renart (1ère branche), CFMA, Champion, Paris.

Rutebeuf, 2001, Euvres complètes, éd. et trad. M. Zink, Lettres Gothiques, Garner, Paris.

SEgre C., 2003, éd. La Chanson de Roland. Tr. M. Tyssens, glos. B. Guidot, Droz, Genève.

ThOmas J., 1989, éd. Renaut de Montauban, TLF, Droz, Genève.

ViLlon F., 1991, Euvres, éd. et trad. A. Lanly, Champion, Paris.

\section{Autres}

BAlZaC H. de (1955), Le Père Goriot, Garnier, Paris.

Voltaire (1959), Candide ou l'optimisme, éd.R. Pomeau, Nizet, Paris.

\section{LATIN}

FLOBERT P., 2000 Le grand Gaffiot, dictionnaire latin-français, Hachette, Paris.

\section{ANGLAIS \\ linguistique}

AdamczewsKi H., 1990, Grammaire linguistique de l'anglais, Armand Colin, Paris.

HewIngs M., 2009, Advanced Grammar in use (A self-study reference and practice book for advanced learners of English), Cambridge University Press. 9ème edition.

\section{Oeuvres citées}

JORDAN R., 2001, The Eye of the world, 17ème éd.depuis 1990, vol 1 des 13 vol. de The Wheel of time. Orbit, Londres.

O’Brian P., 2003, Blue at the Mizzen, vol. 20, HarperCollins, Londres. 